\title{
FORMAÇÃO DE MICELAS MISTAS ENTRE O SAL BILIAR COLATO DE SÓDIO E O SURFACTANTE ANIÔNICO DODECANOATO DE SÓDIO
}

\author{
Renato Eising*, Silvane Morés, Ismael C. Bellettini, Arlindo C. Felippe, Alexandre G. Dal-Bó e Dino Zanette \\ Departamento de Química, Universidade Federal de Santa Catarina, 88040-900 Florianópolis - SC, Brasil
}

Recebido em 26/2/08; aceito em 20/6/08; publicado na web em 31/10/08

\begin{abstract}
MIXED MICELLES FORMATION BETWEEN BILE SALT SODIUM CHOLATE AND THE ANIONIC SURFACTANT SODIUM DODECANOATE. Mixed-micelle formation between sodium chlolate $(\mathrm{NaC})$ and the anionic surfactant sodium dodecanoate (SDoD) in Tris- $\mathrm{HCl}$ buffer solutions, $\mathrm{pH}$ 9.00, varying the molar fraction of the surfactants, was investigated by means of electrical conductivity and steady-state fluorescence of pyrene. The critical micelar concentration $(\mathrm{cmc})$ was measured from the equivalent conductance versus the square root of the molar surfactant concentration plots and the regular solution theory (RST) was used to predict the mixing behavior. The $I_{1} / I_{3}$ pyrene ratio-surfactant concentration plots were used as an additional technique to follow the behavior and the changes in the micropolarity of the mixed micelles.
\end{abstract}

Keywords: electrical conductivity; pyrene fluorescence; $\mathrm{NaC}-\mathrm{SDoD}$ mixed micelles.

\section{INTRODUÇÃO}

Sistemas constituídos de misturas de surfactantes são utilizados em muitas aplicações práticas devido ao baixo custo, comparados àqueles com surfactantes puros, além de favorecer a formação de materiais com propriedades físico-químicas superiores do que quando aplicados individualmente. Do ponto de vista teórico, é relevante o entendimento da formação estrutural e termodinâmica de micelas mistas porque estas estão presentes em processos práticos e tecnológicos, tais como em detergência, emulsões e na recuperação terciária do petróleo..$^{1-4}$

Em solução aquosa, a distribuição de cada surfactante na fase micelar e na fase monomérica varia de acordo com a estrutura do surfactante e com a composição total da solução. A formação de agregados micelares mistos mais simples é aquela quando ocorre sem interações resultantes entre as espécies de surfactantes micelizados. É o caso quando dois diferentes surfactantes são de mesma classe, como misturas de catiônico com catiônico ou de aniônico com aniônico, por exemplo, porém com cadeias hidrofóbicas diferentes. Neste caso, eles possuem propriedades que podem ser previstas a partir de propriedades dos componentes individuais. Estas misturas são denominadas de sistema ideal.

Considerando a micela como uma "fase condensada" em equilíbrio com seu monômero dissociado, a "fase gasosa", no tratamento teórico, a concentração micelar crítica $(\mathrm{cmc})$ é considerada análoga à pressão de vapor do sistema em análise. Este tratamento é denominado de modelo da pseudofase. A $\mathrm{cmc}$, então, está para o equilíbrio monômero-micela assim como a pressão de vapor está para o equilíbrio líquido-vapor. Neste caso, a $\mathrm{cmc}$ é intermediária aos valores de cmc de cada surfactante puro, assim como no processo de variação da pressão de vapor de uma mistura ideal de líquidos. ${ }^{2}$

Para misturas que formam sistemas não-ideais, o tratamento matemático torna-se mais complexo. Exemplos de misturas nãoideais, já bastante estudadas, são as constituídas de surfactantes de diferentes classes, hidrofílicas e/ou hidrofóbicas, tais como misturas de catiônicos com aniônicos ou cada um destes com surfactantes não iônicos. ${ }^{5-7}$ Para estes sistemas não-ideais, os conceitos do modelo da

*e-mail: reising100@yahoo.com.br pseudofase também pode ser aplicados mas, neste caso, são usadas as equações da teoria de soluções regulares ${ }^{3}$ onde são consideradas as interações entre monômeros. Assim, o modelo requer parâmetros empíricos para predizer comportamentos observados. As misturas não-ideais geralmente mostram desvios negativos da idealidade.

Sais biliares são biossurfactantes sintetizados no fígado via oxidação do colesterol e são transportados através da bílis para o intestino. Suas funções estão relacionadas com a solubilização e emulsificação de gorduras e lipídeos. Em humanos, a produção diária de sais biliares, está em torno de 20 g. ${ }^{8}$

Quando dispersados em fase aquosa, os sais biliares podem formar uma variedade de agregados, tipicamente estruturas micelares, que dependem de fatores experimentais, como concentração, natureza química, $\mathrm{pH}$ e temperatura. Micelas de sais biliares são capazes de ligar lipídeos, moléculas anfifílicas e proteínas, formando micelas mistas. Esta habilidade de solubilização é de fundamental importância na absorção intestinal dos produtos da digestão de gorduras como ácidos graxos e monoglicerídios e das vitaminas. ${ }^{9}$

Em água, têm sido extensivamente investigados os processos de micelização dos sais biliares e as conseqüentes estruturas. ${ }^{10-17}$ Small ${ }^{18}$ propôs a formação de micelas primárias e secundárias. No modelo proposto, sugere que na $\mathrm{cmc}$ são formadas somente micelas primárias com agregados com até 10 monômeros, presumivelmente via interações hidrofóbicas entre as partes apolares dos monômeros. Com o aumento da concentração, são formadas micelas secundárias via ligações de hidrogênio dos grupos hidroxilas localizados na superfície externa das micelas primárias.

No entanto, por causa da particular estrutura molecular dos sais biliares, isto é, formada por um esqueleto de esteróide que consiste de uma estrutura rígida com uma face hidrofílica e a oposta hidrofóbica, os processos de associação e agregação diferem consideravelmente daqueles dos surfactantes aniônicos clássicos. ${ }^{19}$ Acrescenta-se, ainda, o fato de terem menor atividade superficial e, ao contrário do seu efetivo comportamento micelar no processo digestivo, sabe-se que, em água, a capacidade de auto-associar é fracamente cooperativa. ${ }^{19,20}$

Neste trabalho, acompanhamos a formação de micelas mistas do sal biliar colato de sódio $(\mathrm{NaC})$ com o aniônico dodecanoato de sódio (SDoD), este último é um surfactante clássico obtido via hidrólise de ácidos graxos e, portanto, também um biossurfactante. A meto- 
dologia empregada para acompanhar os processos de micelização está baseada em gráficos de condutividade elétrica $v s$. concentração dos surfactantes, onde foram obtidos parâmetros como concentração micelar crítica e concentração de pré-agregação (cpa). Baseando-se na teoria da solução regular (RST), o processo de micelização das misturas de $\mathrm{NaC}$ e SDoD foi tratado segundo Rubingh et al.. ${ }^{2,3}$ Através de medidas de fluorescência do pireno, foi utilizada a técnica da razão $\mathrm{I}_{1} / \mathrm{I}_{3}$ das bandas de emissão do pireno $v s$ a concentração da mistura dos surfactantes, para caracterizar as transições no processo de formação dos complexos mistos.

\section{PARTE EXPERIMENTAL}

O colato de sódio (99\%), procedente da Sigma, foi utilizado sem nenhum tratamento prévio. O dodecanoato de sódio foi preparado por neutralização do ácido dodecanóico (Sigma 99\%), conforme o seguinte procedimento: a uma quantidade do ácido carboxílico dissolvido num volume de etanol, sob agitação, adicionava-se solução diluída de hidróxido de sódio mantendo a solução resultante ligeiramente aquecida. A neutralização era controlada por meio de um pHmetro da Beckman modelo $\Phi 71$, equipado com um eletrodo combinado de vidro. A titulação foi conduzida até pH 9,00. Resfriando-se a mistura, o dodecanoato de sódio formava um precipitado pastoso que era filtrado e recristalizado. ${ }^{21}$

As purezas do colato de sódio e do dodecanoato de sódio foram testadas por tensão superficial utilizando, como critério, ${ }^{22}$ a obtenção de um gráfico de tensão superficial vs. logaritmo da concentração molar do surfactante, sem mínimo na região de concentração de surfactante onde ocorre a $\mathrm{cmc}$. Nas condições experimentais estudadas, os valores de $\mathrm{cmc}$ são 25,0 e 10,0 mmol L-1 para o SDoD e o NaC, respectivamente.

Todos os experimentos foram realizados em solução tampão, $\mathrm{pH}$ 9,00, de tris(hidroximetil)aminometano (Tris/HCl) (Aldrich 99\%). As soluções estoques de $20 \mathrm{mmol} \mathrm{L}^{-1}$ de Tris/ $\mathrm{HCl}$ foram preparadas titulando a solução de Tris com solução padrão de $\mathrm{HCl}$, a $25^{\circ} \mathrm{C}$, acompanhando a variação do $\mathrm{pH}$ com o pHmetro da Beckman.

Neste texto, a concentração molar será indicada por colchetes, [ ]. As soluções estoques dos surfactantes foram preparadas pela dissolução de quantidades apropriadas na mesma solução tampão. A água utilizada para os experimentos de condutividade elétrica foi previamente destilada e desmineralizada pelo sistema de purificação de água Milipore (Mili-Q).

\section{Condutividade elétrica}

Nas montagens dos gráficos de condutividade específica versus concentração de surfactante, as medidas de condutividade foram assim executadas: utilizando um condutivímetro da ATI ORION, modelo 170, por meio de uma bureta semi-automática da Methron, modelo E-485, volumes de solução estoque de surfactante eram adicionados sobre um volume conhecido de solução Tris/HCl contido numa cela de diluição, previamente termostatizada a $25,0 \pm 0,1^{\circ} \mathrm{C}$. Com um eletrodo de platina de constante da cela igual a 1,0, a cada adição da solução estoque, fez-se a leitura da condutividade específica da solução resultante. A concentração de surfactante na cela era corrigida em cada adição e leitura de condutividade, considerando o volume total da solução.

\section{Fluorescência}

As medidas de fluorescência do estado estacionário do pireno (Aldrich, 99\%) foram realizadas em soluções tamponadas com 20,0 mmol L ${ }^{-1}$ de Tris/HCl. A solução estoque de pireno, $1,0 \mathrm{mmol} \mathrm{L}^{-1}$, foi preparada em etanol absoluto. A partir da solução estoque, foi preparada em tampão, a solução $1,0 \times 10^{-3} \mathrm{mmol} \mathrm{L}^{-1}$, adicionando-se $1 \mathrm{~mL}$ da solução estoque para cada litro de solução tampão. Em seguida, as soluções estoques de $\mathrm{SDoD}$, de $\mathrm{NaC}$ e das misturas de $\mathrm{SDoD}$, de $\mathrm{NaC}$, foram preparadas com a solução tampão contendo pireno. Os espectros de emissão foram registrados após $6 \mathrm{~h}$ da preparação das soluções minimizando, assim, possíveis erros de instabilidade da intensidade da emissão que ocorre em metodologias alternativas.

O espectro de emissão de fluorescência foi obtido num espectrofluorímetro da Hitachi F4500 equipado com uma cela termostatizada, a 25 ${ }^{\circ} \mathrm{C}$, enquanto a solução de leitura, contida numa cela de quartzo com 10 $\mathrm{mm}$ de caminho óptico, era levemente agitada. As fendas, de excitação e emissão, foram ajustadas em 2,5 nm. A excitação das amostras ocorria em 336,0 nm e as emissões foram registradas no intervalo de 360,0 a $500,0 \mathrm{~nm}$. A razão $\mathrm{I}_{1} / \mathrm{I}_{3}$ foi estimada medindo as intensidades dos picos em 372,8 nm ( $\left.I_{1}\right)$ e 384,0 (I $\left.I_{3}\right)$ do espectro de emissão do pireno.

\section{RESULTADOS E DISCUSSÃO}

\section{Condutividade elétrica}

A condutividade elétrica é a medida da mobilidade de espécies iônicas em solução. Numa solução ela é dependente da concentração e dos eletrólitos presentes. No caso de eletrólitos fortes, a variação na condutividade elétrica da solução é diretamente proporcional à concentração de eletrólito adicionado. Já para fracos, como a concentração de íons é dependente do equilíbrio de dissociação, a condutividade dependerá do grau de ionização $(\alpha)$ do eletrólito. Micelas iônicas são agregados que podem ligar ânions e/ou cátions de tal forma que, na $\mathrm{cmc}$, o conteúdo iônico da solução varia com a formação delas e, o gráfico de condutividade elétrica $v s$ concentração do surfactante, na $c m c$, mostra descontinuidade. Uma informação adicional obtida dos gráficos de condutividade refere-se aos coeficientes angulares das regiões lineares do gráfico que, abaixo e acima da $c m c$, estão relacionados com o conteúdo iônico da solução. Numa aproximação, a razão entre os coeficientes angulares está relacionada com o grau de ionização micelar. ${ }^{19}$

A Figura 1 mostra as mudanças da condutividade específica do $\mathrm{SDoD}$ e do $\mathrm{NaC}$, e de misturas. Para o SDoD, o perfil exibe uma $\mathrm{cmc}$ em $25,0 \mathrm{mmol} \mathrm{L}^{-1}$ e uma pequena inflexão $\approx 5,0 \mathrm{mmol} \mathrm{L}^{-1}$ (Figura 2a) evidenciada pela diferença dos coeficientes angulares acima e abaixo deste ponto de descontinuidade (Tabela 1). Esta mudança

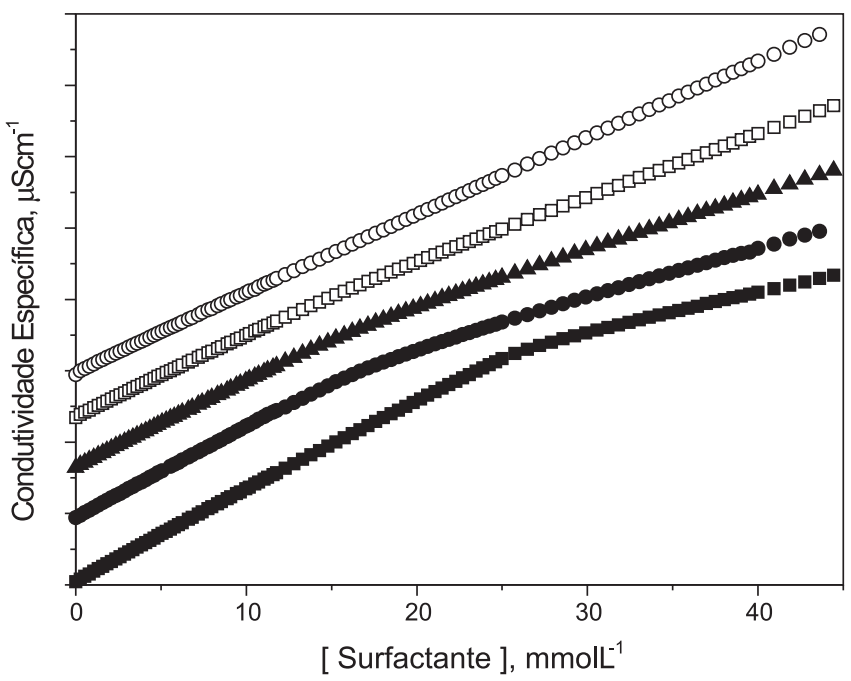

Figura 1. Gráficos de condutividade especifica vs. concentração total de surfactante, em várias frações molares de SDoD: (回 1,$0 ;(\mathbf{O}) 0,8 ;(\mathbf{\Delta})$ 0,6 ; ( $\square$ ) 0,4 ; (О) 0,0 

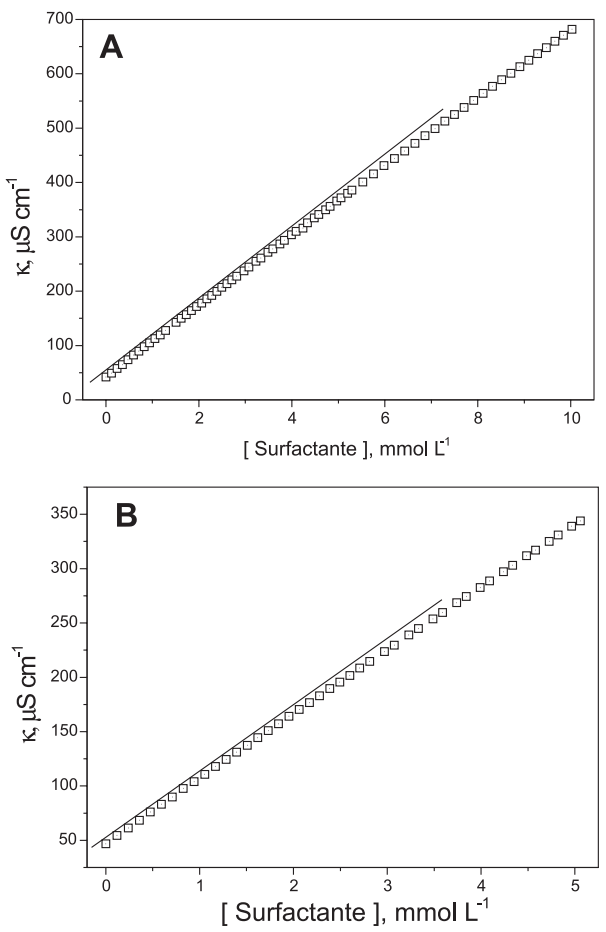

Figura 2. Gráficos de condutividade especifica vs. concentração de SDoD (a) e de $\mathrm{NaC}$ (b) obtidos na região de pré-agregação

de inclinação é atribuída à capacidade do SDoD em formar préagregados. Este comportamento já tem sido identificado em reações catalisadas por SDoD. ${ }^{23}$

No entanto, em concordância com resultados anteriores, ${ }^{20,24}$ observa-se na Figura 1 a ausência de um ponto de descontinuidade equivalente da $\mathrm{cmc}$. Na verdade, este perfil é consistente com o mecanismo de micelização de sais biliares que se caracterizam por um processo de agregação progressiva, inicialmente formando micelas pequenas nas proximidades da $\mathrm{cmc}$, mas crescendo com o aumento da concentração biliar (para maiores detalhes ver refs. 10, 17, 20 e 24). Observa-se na Figura $2 b$, no entanto, uma pequena inflexão em concentrações abaixo daquela prevista para a $\mathrm{cmc}$ do $\mathrm{NaC}$. Uma leve inflexão em 2,5 mmol L-1 é indicada como a concentração de formação de dímeros. ${ }^{25}$ Este fato é consistente com resultados de Oakenfull e Fisher, ${ }^{12}$ onde o desvio observado no perfil de condutividade molar no gráfico da Equação de Onsager foi atribuído à formação de dímeros.
Do mesmo modo, misturas de SDoD e NaC apresentaram o mesmo comportamento em todas as frações molares estudadas.

Com a adição de $\mathrm{SDoD}$ à solução contendo $\mathrm{NaC}$, os perfis tendem a exibir descontinuidades (Figura 1). Neste trabalho, a determinação da $c m c$ das misturas foi melhor executada graficando os resultados de condutividade na forma de condutividade equivalente $v s$. raiz quadrada de [surfactante] (Figura 3b). Os valores de $c m c$ em função da fração molar estão listados na Tabela 1.

Apesar dos perfis de condutividade especifica não serem os mais adequados para a determinação da $c m c$, ainda são bastante úteis no estudo das misturas SDoD-NaC. A partir destes perfis, pode-se obter os valores dos coeficientes angulares das regiões lineares e, portanto, estimar o grau de ionização micelar $(\alpha)$ mediante a razão dos coeficientes angulares (Tabela 1) que, embora em outras condições experimentais, são semelhantes àqueles obtidos por Zanette e Frescura. ${ }^{26}$ Para o $\mathrm{NaC}$, o valor de $\alpha \approx 1$ representa uma estrutura micelar que não tem a capacidade de ligar contra-íons.

Assim como para os surfactantes puros, em misturas deles também foram observadas pequenas descontinuidades em concentrações entre 2,5 e 5,0 mmol L-1 (Tabela 1). Para definir a formação de pré-agregados com mais exatidão, foram realizadas medidas de condutividade elétrica em concentrações diluídas. Os valores de cpa estão listados na Tabela 1. A formação e composição destes pré-agregados estão relacionadas com a fração molar e concentração total de surfactante.

\section{Modelo de micelização de mistura de SDoD e NaC}

Numa mistura binária formada pelos surfactantes A e B, a cmc da mistura de um sistema ideal pode ser determinada pela fração molar de um dos componentes da mistura e a $\mathrm{cmc}$ dos surfactantes individuais, conforme mostra a Equação 1, ${ }^{1-3}$

$c m c_{\text {ideal }}=\frac{c m c_{A} c m c_{B}}{\chi_{A} c m c_{B}+\chi_{B} c m c_{A}}$

onde, $c m c_{\text {Ideal }}$ é a concentração micelar crítica segundo a teoria da solução ideal, $c m c_{\mathrm{A}}$ e $c m c_{\mathrm{B}}$ referem-se às $c m c$ dos surfactantes A e $\mathrm{B}$, respectivamente, e $\chi_{A}$ e $\chi_{B}$ são as frações molares dos surfactantes A e B na solução, respectivamente.

Para misturas que formam sistemas não-ideais, sendo geralmente misturas de surfactantes constituídos por classes hidrofílicas ou hidrofóbicas, tais como misturas de catiônicos com aniônicos ou cada um destes com surfactantes não iônicos, o tratamento matemático

Tabela 1. Valores de concentração micelar crítica $(\mathrm{cmc})$, concentração de agregação crítica $(\mathrm{cpa})$, e dos coeficientes angulares obtidos a partir de gráficos de condutividade elétrica

\begin{tabular}{|c|c|c|c|c|c|c|}
\hline$\chi_{\mathrm{SDoD}}$ & $c m c \mathrm{mmol} \mathrm{L}^{-1}$ & cpa $\mathrm{mmol} \mathrm{L}^{-1}$ & $\mathrm{~S}_{11}$ & $\mathrm{~S}_{12}$ & $\mathrm{~S}_{3}$ & $\alpha$ \\
\hline 1,0 & 25,0 & 5,1 & 66,6 & 63,2 & 27,4 & 0,41 \\
\hline 0,9 & 18,0 & & 66,6 & 62,0 & 31,9 & 0,48 \\
\hline 0,8 & 15,6 & 4,4 & 65,2 & 63,2 & 33,5 & 0,51 \\
\hline 0,7 & 14,7 & & 65,5 & 61,1 & 35,1 & 0,54 \\
\hline 0,6 & 14,3 & 3,5 & 63,7 & 59,7 & 38,3 & 0,60 \\
\hline 0,5 & 13,9 & & 59,1 & 56,0 & 41,2 & 0,70 \\
\hline 0,4 & 13,8 & 3,2 & 58,8 & 56,8 & 44,3 & 0,75 \\
\hline 0,3 & 13,3 & & 59,1 & 55,6 & 46,0 & 0,79 \\
\hline 0,2 & 12,4 & 3,0 & 60,0 & 57,7 & 52,7 & 0,88 \\
\hline 0 & 10,0 & 2,6 & 59,5 & 55,8 & 53 & 0,89 \\
\hline
\end{tabular}

Os coeficientes angulares $S_{11}$ e $S_{12}$ referem-se às inclinações acima e abaixo da concentração de pré-agregação e $S_{3}$ refere-se ao coeficiente angular acima da $\mathrm{cmc}$. 

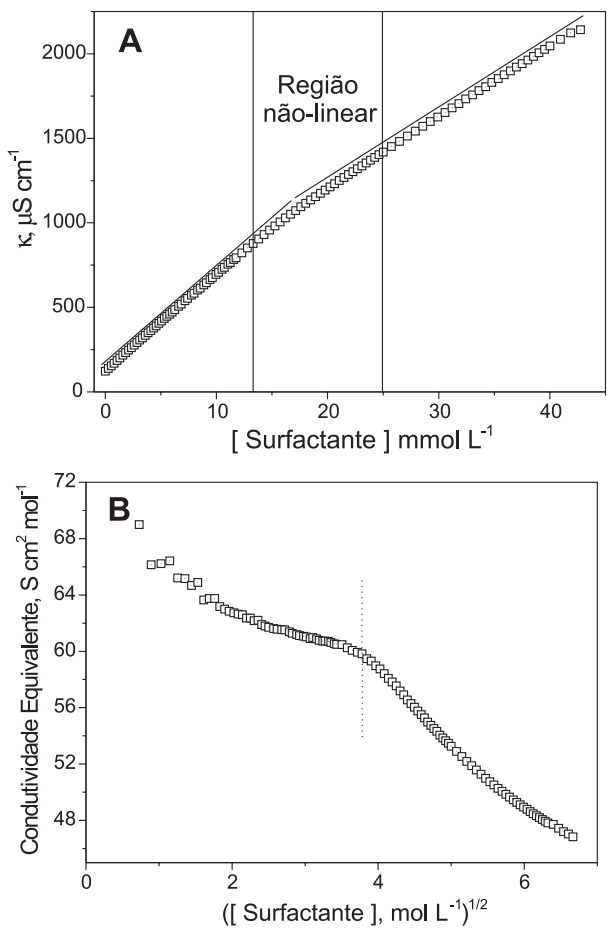

Figura 3. Perfil de condutividade especifica vs [SDoD] (a) e de condutividade equivalente vs. raiz quadrada da [SDoD] (b) em 0,6 de fração molar

torna-se mais complexo. Para estes sistemas não-ideais, o modelo da pseudofase também pode ser aplicado mas, neste caso, são usadas as equações da teoria das soluções regulare ${ }^{1-3} \mathrm{e}$ as interações surfactante/ surfactante devem ser consideradas, assim como os coeficientes de atividade. O modelo requer um parâmetro empírico para predizer o comportamento observado.

Estas misturas geralmente mostram desvios negativos da idealidade. Logo, a $c m c$ observada, é menor que aquela prevista pela Equação 1. Assim, se a interação entre os surfactantes A e B na micela mista pode ser descrita pelo modelo da aproximação regular da solução, a Equação $2^{2,3}$ fornece as informações sobre o comportamento da formação de micelas mistas que apresentam desvios da idealidade.

A Equação 2 prevê a composição da micela mista, ou seja, a fração molar de cada surfactante na micela,

$\chi_{A}^{2} \ln \left(\frac{c m c_{\exp } \chi_{A}}{c m c_{A} x_{A}}\right)=\left(1-x_{A}\right)^{2} \ln \left[\frac{c m c_{\exp }\left(1-\chi_{A}\right)}{c m c_{B}\left(1-x_{A}\right)}\right]$

O processo de micelização das misturas de $\mathrm{SDoD}$ e $\mathrm{NaC}$ pode ser tratado aplicando-se a teoria da aproximação regular da solução (RST), desenvolvida por Rubingh e colaboradores. ${ }^{2,27}$ De acordo com este modelo, os coeficientes de atividade dos surfactantes são relacionados com um parâmetro de interação, $\beta$, que quantifica a interação líquida entre as espécies de surfactantes na micela. Valores positivos de $\beta$ indicam que existe uma repulsão resultante entre os dois surfactantes componentes da micela, enquanto que valores negativos representam um processo de micelização da mistura onde ocorre atração líquida entre as espécies de surfactantes. As linhas contínuas na Figura 4 representam os valores de $\mathrm{cmc}$ das misturas estimados pela teoria da aproximação da solução regular para diferentes valores do parâmetro líquido de interação $\beta$. A linha pontilhada corresponde ao comportamento ideal da solução obtido quando $\beta=0$. O valor médio de $\beta$, encontrado para a mistura SDoD-NaC, foi de $-0,2$ mas, como se pode observar na Figura 4, este valor não representa corretamente os dados de $c m c$ obtidos experimentalmente. O processo de micelização

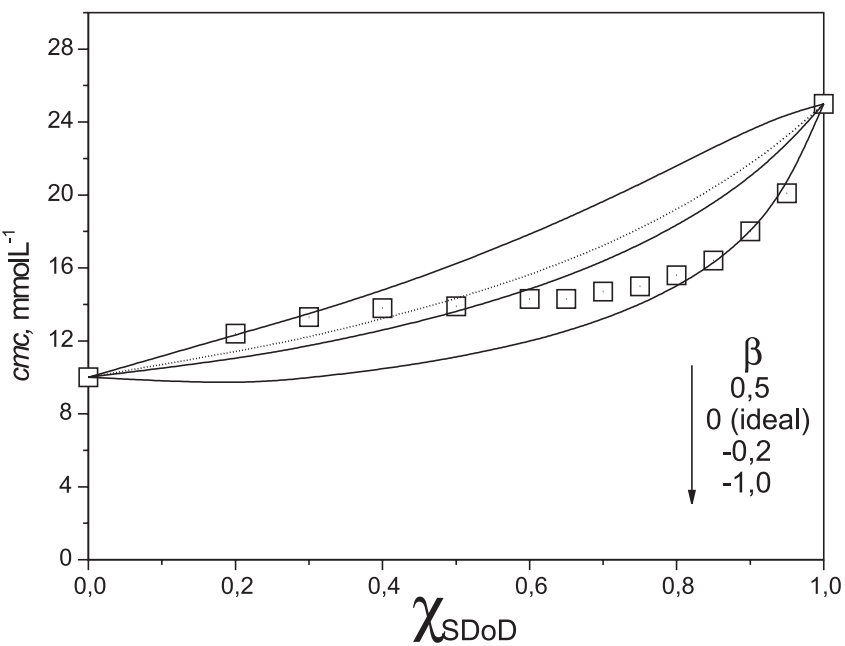

Figura 4. Variação da cmc experimental de misturas de NaC e SDoD ( $\square$ ) segundo a teoria da solução ideal (linha pontilhada) e segundo a teoria da solução regular (RST), para diferentes valores de $\beta$ (linhas contínuas), em função da fração molar de $S D o D$

das misturas de SDoD-NaC mostra um desvio negativo da idealidade para frações molares de $\mathrm{SDoD}>0,5$, enquanto que ocorre um desvio positivo para frações molares de $\mathrm{SDoD}<0,5$. Uma simulação, utilizando um valor de $\beta=-1,0$, mostra-se bastante coerente com os valores experimentais de $\mathrm{cmc}$ obtidos para altas frações molares de $\operatorname{SDoD}\left(\chi_{\mathrm{SDoD}}>0,8\right)$, enquanto é de baixa coerência em altas frações molares de $\mathrm{NaC}$. De outro lado, uma simulação $\operatorname{com} \beta=+0,5$ é satisfatória para baixas frações molares de $\operatorname{SDoD}\left(\chi_{\mathrm{SDoD}}<0,4\right)$, mas o desvio se torna grande com incrementos de $\mathrm{SDoD}$ à mistura. $\beta=-1,0$, representa um sistema com uma baixa atração líquida entre os monômeros, enquanto que, $\beta=+0,5$ representa um sistema com uma baixa repulsão líquida entre estes. Desde que os surfactantes são aniônicos, a interação deve ser exclusivamente hidrofóbica. Observou-se ainda que, nos ajustes, o parâmetro líquido de interação $\beta$ variou de $-1,0$ a 1,0 para as frações molares de SDoD de 0,9 e 0,3, indicando que a formação de micelas ricas em SDoD ocorre com maior atração entre os monômeros que na formação de micelas ricas em $\mathrm{NaC}$.

A Figura 5 mostra a variação da composição das micelas mistas para diferentes misturas de SDoD-NaC em função da fração molar total de surfactante na solução determinadas pelas teorias da solução ideal e aproximação da solução regular. ${ }^{2}$ A linha pontilhada representa a composição micelar na qual não existe nenhuma interação entre os monômeros, ou seja, um comportamento ideal. ${ }^{27}$ Em altas frações molares de $\operatorname{SDoD}\left(\chi_{\mathrm{SDoD}} \approx 0,8\right)$, a teoria prediz uma absorção preferencial de monômeros de SDoD nas micelas mistas e para $\chi_{\mathrm{NaC}}$ $>0,7$ as típicas micelas de $\mathrm{NaC}$ exibem uma composição micelar com preferência para monômeros de $\mathrm{NaC}$. A composição das micelas é igual de acordo com as duas teorias para a fração molar total de SDoD na solução igual a 0,47 indicando uma micela mista com uma composição de $26 \%$ de SDoD (Figura 5). No entanto, de acordo com a teoria da solução regular, observa-se que a quantidade de monômeros de SDoD na micela mista para $\chi_{\mathrm{SDoD}}<0,47$ é inferior àquela prevista pela teoria da solução ideal. Já para $\chi_{\text {SDoD }}>0,47$, a quantidade de monômeros de SDoD na micela mista é superior àquela prevista pela teoria da solução ideal. Ainda, conforme a teoria da aproximação da solução regular, na Figura 5 são destacadas três regiões distintas: primeiro, para frações molares totais de SDoD menores que 0,3 , observa-se uma mudança rápida no perfil da composição das micelas, indicando a formação de micelas tipicamente ricas em $\mathrm{NaC}$. Segundo, em frações molares de SDoD entre 0,3 e 0,8 , a composição varia de 


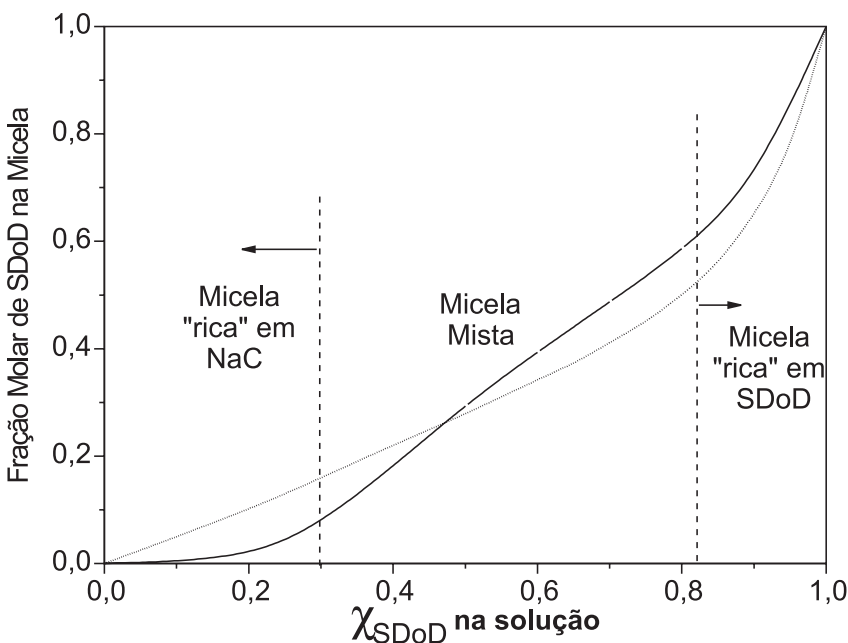

Figura 5. Variação da composição monomérica das micelas mistas segundo a teoria da solução ideal (linha pontilhada) e segundo a teoria da solução regular (linha contínua) em função da fração molar total de SDoD na solução

forma contínua e gradativa, em concordância com um sistema de formação de micelas mistas. Por fim, em frações molares de SDoD superior a 0,8 , observa-se que estão sendo formadas micelas tipicamente ricas em SDoD.

\section{Perfis de $I_{1} / I_{3} v s$. fração molar dos surfactantes}

Recentemente, em nosso laboratório tem-se utilizado o pireno para monitorar as mudanças de polaridade em agregados micelares formados em misturas de polímero e surfactante. ${ }^{24}$ Neste trabalho, em altas concentrações de $\mathrm{SDoD}$, observou-se que a razão $\mathrm{I}_{1} / \mathrm{I}_{3}$ tende a 1,1 , enquanto que, em frações altas de $\mathrm{NaC}$, ela diminui para valores próximos de 0,8 . Esta significativa diferença dos valores de $\mathrm{I}_{1} / \mathrm{I}_{3} \mathrm{em}$ função da fração molar da mistura, qualitativamente, serviu como técnica para acompanhar a variação da composição do agregado misto de SDoD e NaC. A Figura 6 mostra os perfis em diferentes concentrações fixas da mistura, variando as frações molares.

$\mathrm{Na}$ interpretação dos resultados da Figura 6, são levados em consideração dois pontos relevantes: o primeiro refere-se à $\mathrm{cmc}$ de cada fração molar da mistura sendo, neste caso, o diagrama da Figura

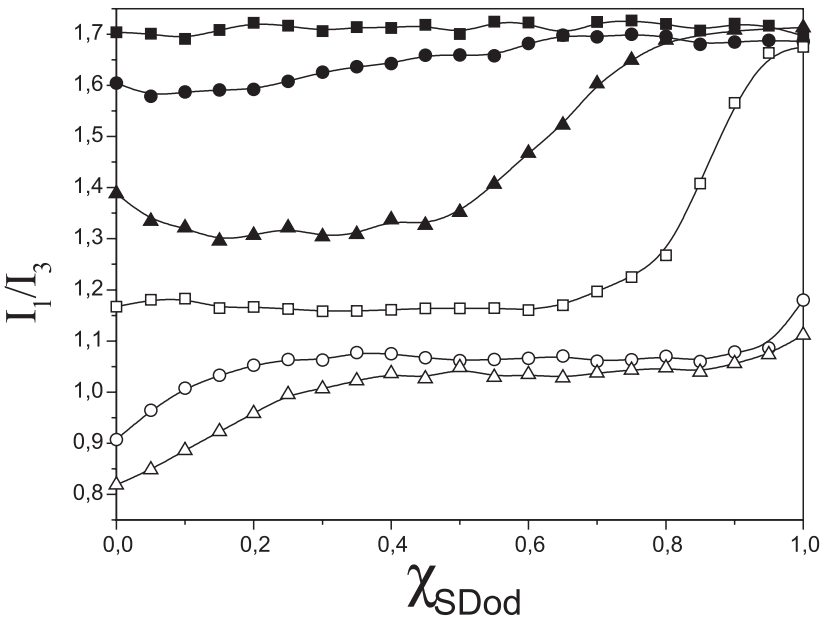

Figura 6. Perfis da razão $I_{1} / I_{3}$ do espectro de fluorescência do pireno em

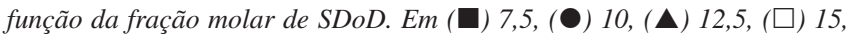

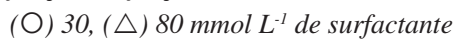

4, uma referência na discussão. A premissa é de que, sempre que a razão $\mathrm{I}_{1} / \mathrm{I}_{3}$ é avaliada próximo da $\mathrm{cmc}$, espera-se que ocorram fortes mudanças de polaridade do pireno. $\mathrm{O}$ segundo ponto a ser considerado, refere-se ao fato de que, mesmo em $\mathrm{NaC}$ puro e quando sua concentração é aumentada, a sonda indica contínuas mudanças de polaridade. Assim, quando na ausência de SDoD, os perfis da Figura 6 indicam que a razão decresce de 1,7, quando em água, a 0,82. Este fato deve estar relacionado com as contínuas variações estruturais das micelas de $\mathrm{NaC}$ que ocorrem quando concentração é aumentada. Recentemente, utilizando sondas derivadas do naftaleno, Bohne et al. ${ }^{28}$ sugeriram a concentração $20,0 \mathrm{mmol} \mathrm{L}^{-1}$ de $\mathrm{NaC}$, como um limite de mudança de predominância de micelas primárias para a das secundárias. Assim, os resultados mostrados na Figura 6, podem ser encarados como mais uma evidência para enfatizar a conclusão de Bohne et al. ${ }^{28}$ considerando que, no perfil com a concentração de surfactante de $15,0 \mathrm{mmol} \mathrm{L}^{-1}$, o valor de $\mathrm{I}_{1} / \mathrm{I}_{3}$ indica que o pireno sente uma polaridade intermediária. Isto é, em concentrações menores, a sonda indica um ambiente mais polar enquanto que, acima, a polaridade tende para valores próximos daqueles de hidrocarbonetos.

A partir dos perfis da Figura 6, anotam-se as seguintes considerações: nas concentrações menores que a $c m c\left(7,5 \mathrm{mmol} \mathrm{L}^{-1}\right)$ e no limite dela $\left(10,0 \mathrm{mmol} \mathrm{L}^{-1}\right)$, os valores de $\mathrm{I}_{1} / \mathrm{I}_{3}$ são semelhantes aos valores em água $\left(I_{1} / I_{3}=1,7\right)$. Neste caso, o pireno indica uma polaridade tipicamente da água porque, em todas as frações molares, os agregados micelares estão ausentes e/ou a concentração de agregados micelares é pequena. Nas várias misturas estudadas, quando em condições de alta concentração de $\mathrm{NaC}$, a polaridade gradativamente diminui com o aumento da [surfactante] porque os agregados micelares são tipicamente agregados de $\mathrm{NaC}$ e comportam-se como acima comentado, quando na ausência de SDoD. Salienta-se que, em 12,5 $\mathrm{mmol} \mathrm{L}^{-1}$ da mistura, portanto menor que a concentração limite de $20,0 \mathrm{mmol} \mathrm{L}^{-1}$ de $\mathrm{NaC}$, com o aumento da $\chi_{\mathrm{SDoD}}$, a razão $\mathrm{I}_{1} / \mathrm{I}_{3}$ ligeiramente diminui, atinge um platô e, a partir de $\chi_{\mathrm{SDoD}} \approx 0,5$, aumenta até atingir valores de $\mathrm{I}_{1} / \mathrm{I}_{3}=1,7$ em altas frações de SDoD. Observa-se que, em $\chi_{\mathrm{SDoD}} \approx 0,5$, a $\mathrm{cmc}$ da mistura é próxima de $14 \mathrm{mmol} \mathrm{L}^{-1}$ (Figura 4) o que justifica o pireno localizado em microambientes de polaridade gradativamente alta.

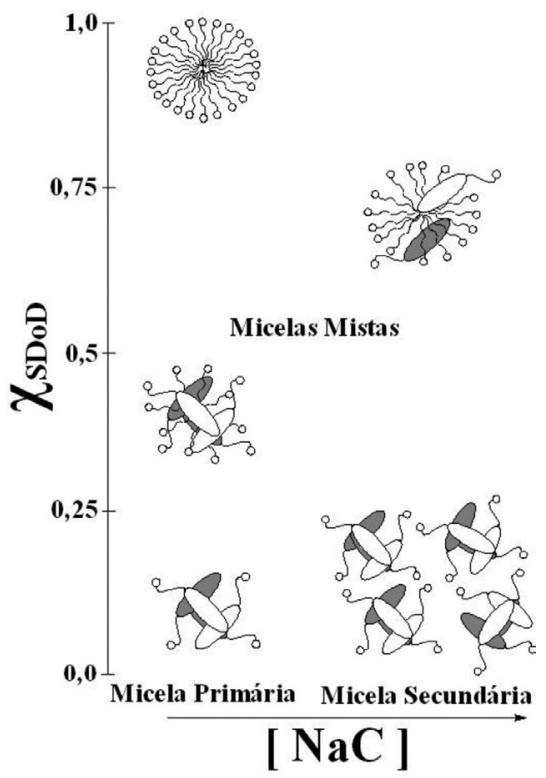

Figura 7. Modelo estrutural de micelas mistas de NaC e de SDoD em diferentes composições. Em frações ricas em $\mathrm{NaC}$ a formação de agregados de forma progressiva (micelas primárias e secundárias); em frações intermediárias a formação de micelas mistas de NaC-SDoD e em frações ricas de $S D o D$ a formação de agregados tipicamente de SDoD 
Em concentrações da mistura maiores que $20,0 \mathrm{mmol} \mathrm{L}^{-1}$, portanto, em 30 e $80 \mathrm{mmol} \mathrm{L}^{-1}$, a razão $\mathrm{I}_{1} / \mathrm{I}_{3}$ aumenta e, a partir de $\chi_{\mathrm{SDoD}}$ $\approx 0,2$ inicia um platô que se estende até $\approx 0,8 \mathrm{e}$, acima, os valores de $\mathrm{I}_{1} / \mathrm{I}_{3}$ são tipicamente de micelas de $\mathrm{SDoD}, \mathrm{I}_{1} / \mathrm{I}_{3}=1,10 \mathrm{em} 80 \mathrm{mmol}$ $\mathrm{L}^{-1}$. (Lit. $\left.=1,04\right) \cdot{ }^{29}$

\section{CONCLUSÃO}

Embora o sistema NaC-SDoD seja composto por agregados micelares de estruturas e composições diferentes, basicamente, duas variáveis são predominantes na polidispersidade do sistema, isto é, concentração do colato de sódio e fração molar da mistura. Como todo sistema binário de mistura de surfactantes, ${ }^{3,5-7}$ aqui também se observa que, em altas frações dos surfactantes, predominam micelas tipicamente de SDoD e/ou de $\mathrm{NaC}$. Este fato é evidenciado pela Figura 5, relacionado com as rápidas mudanças no perfil da composição das micelas em frações molares de SDoD acima de 0,8 e em menores que 0,3. Estas indicações de mudanças estruturais da mistura são também mostradas pelas bruscas variações de polaridade indicadas nos perfis de $\mathrm{I}_{1} / \mathrm{I}_{3} v s \chi_{\mathrm{SDoD}}$ da Figura 6. Já em frações molares compreendidas entre 0,3 e 0,8 , os perfis $\mathrm{I}_{1} / \mathrm{I}_{3}$ vs $\chi_{\mathrm{SDoD}}$ mostram que a polaridade pouco varia, indicando o desenvolvimento de agregados micelares de estruturas idênticas. Como é visto na Figura 5, a composição nesta fase é caracterizada por uma variação contínua e gradativa em concordância com um sistema de formação de micelas mistas que, no caso, são de $\mathrm{NaC}$ e SDoD. Um modelo micelar proposto para a variação da composição da mistura é exemplificado pela Figura 7.

\section{REFERÊNCIAS}

1. Scamehorn, J. F. Em Phenoma in Mixed Surfactant Systems - ACS Symposium Series 311; Scamehorn, J. F., ed.; American Chemical Society: Washington, 1986, cap. 1.

2. Holland, P. M.; Rubingh, D. N. Em Mixed Surfactants Systems - ACS Symposium Series 501; Holland, P. M.; Rubingh, D. N., eds.; American Chemical Society: Washington, 1992, cap. 1

3. Holland, P. M.; Rubingh, D. N.; J. Phys. Chem. 1983, 87, 1984.

4. Nitschke, M.; Pastore, G. M.; Quim. Nova 2002, 25, 772.

5. Hierrezuelo, J. M.; Aguiar, J.; Ruiz, C. C.; Colloids Surf., A 2005, 264, 29.
6. Joshi, T.; Mata, J.; Bahadur, P.; Colloids Surf., A 2005, 260, 269.

7. Hoffmann, H.; Possneckert, G.; Langmuir 1994, 10, 381.

8. Fabry, B.; Chemie in Unserer Zeit 1991, 25, 214.(CA 116:108807).

9. Gouin, S.; Zhu, X. X.; Langmuir 1998, 14, 4025.

10. Small, D. M.; Penkett, S. A.; Biochim. Biophys. Acta 1969, 176, 178.

11. Barry, B. W.; Gray, G. M.; J. Colloid Interface Sci. 1975, 52, 314.

12. Oakenfull, D. G.; Fisher, L. R.; J. Phys. Chem. 1977, 81, 1838.

13. Lang, J.; Zana, R. Em Surfactant Solutions: New Methods of Investigation; Zana, R., ed.; Marcel Dekker: New York, 1987, cap. 4.

14. Djavanbakht, A.; Kale, K. M.; Zana, R.; J. Colloid Interface Sci. 1977, $59,139$.

15. Kratohvil, J. P.; Hsu, W. P.; Kwok, D. I.; Langmuir 1986, 2, 256.

16. Coello, A.; Meijide, F.; N nez, R.; Tato, J. V.; J. Phys. Chem.1993, 97 , 10186.

17. Hildebrand, A.; Garidel, P.; Neubert, R.; Blume, A.; Langmuir 2004, 20, 320.

18. Small, D. M.; Advances in Chemistry Series 1968, 84, 31.

19. Zanette, D.; Schweitzer, B.; Felippe, A. C.; Dal Bó, A.; Lopes, A.; Colloids Surf., A 2006, 279, 87.

20. Ueno, M.; Kimono, Y.; Ikeda, I.; Momose, H.; Zana, R.; J. Colloid Interface Sci. 1987, 177, 179.

21. Perrin, D. D.; Armarego, W. L. F.; Purification of Laboratory Chemicals, $3^{\text {rd }}$ ed., Pergamon Press: New York, 1988.

22. Shaw, D. J.; Introdução à Química de colóides e de Superfícies, Edgard Blücher: São Paulo, 1975.

23. Marconi, D. M. O.; Frescura, V. L.; Zanette, D.; Nome, F.; J. Phys. Chem. 1994, 98, 12415.

24. Felippe, A. C.; Schweitzer, B.; Dal Bó, A.; Eising, R.; Minatti, E.; Zanette, D.; Colloids Surf., A 2007, 294, 247.

25. Felippe, A. C.; Dissertação de mestrado, Universidade Federal de Santa Catarina, Brasil, 2006

26. Zanette, D.; Frescura, V.; Colloids Surf. 1999, 213, 379.

27. Holmberg, K.; Jonsson, B.; Kronberg, B.; Lindman, B.; Surfactants and Polymers in Aqueous Solution, John Wiley \& Sons Ltda: Chichester, 2004.

28. Bohne, C.; Rinco, O.; Nolet, M. C.; Ovans, R.; Photochem. Photobiol. Sc. 2003, 2, 1140.

29. Kalyanasundaran, K.; Thomas, J. K.; J. Am. Chem. Soc. 1977, 99, 2039. 